OPEN ACCESS

Edited by:

Junji Xing,

Houston Methodist Research Institute,

United States

Reviewed by:

Renfeng $\mathrm{Li}$,

Virginia Commonwealth University,

United States

Zongdi Feng,

The Ohio State University,

United States

${ }^{*}$ Correspondence:

Hinh Ly

hly@umn.edu

${ }^{\dagger}$ These authors share senior authorship

Specialty section:

This article was submitted to Molecular Innate Immunity, a section of the journal

Frontiers in Immunology

Received: 25 October 2021 Accepted: 17 November 2021 Published: 03 December 2021

Citation:

Brisse M, Huang Q, Rahman M, Di D, Liang Y and Ly H (2021) R/G-I and MDA5 Protect Mice From Pichinde

Virus Infection by Controlling Viral Replication and Regulating Immune

Responses to the Infection.

Front. Immunol. 12:801811.

doi: 10.3389/fimmu.2021.801811

\section{RIG-I and MDA5 Protect Mice From Pichinde Virus Infection by Controlling Viral Replication and Regulating Immune Responses to the Infection}

\author{
Morgan Brisse ${ }^{1,2}$, Qinfeng Huang ${ }^{2}$, Mizanur Rahman $^{2}$, Da Di $^{2}$, Yuying Liang ${ }^{2 \dagger}$ \\ and Hinh $L y^{1,2 * t}$ \\ 1 Biochemistry, Molecular Biology and Biophysics Graduate Program, College of Veterinary Medicine, University of \\ Minnesota, Twin Cities, MN, United States, 2 Department of Veterinary and Biomedical Sciences, College of Veterinary \\ Medicine, University of Minnesota, Twin Cities, MN, United States
}

RIG-I and MDA5 are major cytoplasmic innate-immune sensor proteins that recognize aberrant double-stranded RNAs generated during virus infection to activate type 1 interferon (IFN-I) and IFN-stimulated gene (ISG) expressions to control virus infection. The roles of RIG-I and MDA5 in controlling replication of Pichinde virus (PICV), a mammarenavirus, in mice have not been examined. Here, we showed that MDA5 single knockout (SKO) and RIG-I/MDA5 double knockout (DKO) mice are highly susceptible to PICV infection as evidenced by their significant reduction in body weights during the course of the infection, validating the important roles of these innate-immune sensor proteins in controlling PICV infection. Compared to the wildtype mice, SKO and DKO mice infected with PICV had significantly higher virus titers and lower IFN-I expressions early in the infection but appeared to exhibit a late and heightened level of adaptive immune responses to clear the infection. When a recombinant rPICV mutant virus (rPICV-NPmut) that lacks the ability to suppress IFN-I was used to infect mice, as expected, there were heightened levels of IFN-I and ISG expressions in the wild-type mice, whereas infected SKO and DKO mice showed delayed mouse growth kinetics and relatively low, delayed, and transient levels of innate and adaptive immune responses to this viral infection. Taken together, our data suggest that PICV infection triggers activation of immune sensors that include but might not be necessarily limited to RIG-I and MDA5 to stimulate effective innate and adaptive immune responses to control virus infection in mice.

Keywords: Pichinde virus, mammarenavirus, arenavirus, innate immunity, RIG-I, MDA5, nucleoprotein 


\section{INTRODUCTION}

Mammarenaviruses are primarily rodent borne viruses, which are found in West Africa and South and Central America (1). Several members of this Arenaviridae family can cause hemorrhagic fever diseases in humans with limited options for treatment and vaccination (2-6). Lassa virus (LASV), which is an Old World mammarenavirus that causes endemic Lassa fever in several West African countries has been designated by the World Health Organization (WHO) among the blueprint priority pathogens that need the highest priority for research and development (7). Mammarenaviruses are transmitted via human contact with contaminated food and water or excreta from locally infected rodent populations [e.g., Mastomys natalensis $(8,9)]$ that do not appear to experience severe disease symptoms; and they therefore act as viral reservoirs (10-12).

There are currently no effective anti-viral treatments for Lassa fever other than the use of the non-specific anti-viral ribavirin, which must be given in the early stages of the disease to demonstrate efficacy (13). Except for the Candid \#1 vaccine that has only been approved for use in Argentina to control human infections by the New World Junin virus (NW JUNV), there are currently no available vaccines for other pathogenic human mammarenaviruses. A notable factor in humans that has been shown to be associated with the development of severe disease due to LASV infection is the lack of the development of the proinflammatory cytokine responses in the early phase of the infection (14-17). Type 1 interferons (IFN-I), which consists of IFN $\alpha$ and $\operatorname{IFN} \beta(18,19)$, are critical cytokines needed to control the early phase of virus replication and to help initiate cellular inflammation as well as adaptive immune responses. Specifically, IFN-I expressions help drive the expression of hundreds of interferon-stimulated genes (ISGs) that play critical antiviral roles and aid the activation of $\mathrm{T}$ and $\mathrm{B}$ cells to produce cellular (helper $\mathrm{CD} 4$ and cytotoxic CD8 T cells) and humoral (antibody) responses against the virus infection (20). IFN-Is are expressed when the host RIG-I like Receptor (RLR) cellular proteins, which consist of RIG-I and MDA5, are activated upon recognizing pathogen-associated molecular pattern dsRNAs (PAMP dsRNAs) that are aberrantly generated during the process of viral RNA replication to activate the RLR signaling cascade to produce IFN-I $(21,22)$. While a significant amount of work has been done to elucidate the unique roles of RIG-I and MDA5 and their individual importance in antiviral defense (23), the overall and relative importance of each factor against mammarenaviral infection is not clear.

Pichinde virus (PICV), which is a NW BSL2-level mammarenavirus that normally does not cause disease in humans or mice but has been adapted for use in guinea pigs as a surrogate model of Lassa fever $(24,25)$. It adopts a life cycle like that of other mammarenaviruses. Virus particles enter cells by clathrin-mediated endocytosis (26), though the primary cellular receptor has not been identified for PICV (6). Replication occurs in the cytosol when the RNA-dependent RNA polymerase (L) in complex with the nucleoprotein (NP) synthesizes both anti-genomic and genomic strands of viral RNA, which is necessary for both viral replication and for viral gene expression (27). NP also has exoribonuclease (ExoN) functionality that is thought to degrade viral RNA to avoid detection by innate immune receptors, such as RIG-I and
MDA5 (28). Formation and budding of progeny virus particles are aided by the viral $\mathrm{Z}$ matrix protein (29). While the host tropism of PICV has not been fully determined, the virus has been shown to infect both guinea pigs and mice as well as cells from several other species, including African green monkey (Vero) (30), hamster (BHK21) (30) and human (PBMCs and THP1) (31). While the tissue tropism within mice and guinea pigs can be quite diverse, infectious virus and pathology mediated by virus infection can be found systemically in guinea pigs (25) and suckling mice $(32,33)$.

In the current study, we infected mice deficient for MDA5 alone (SKO) or both RIG-I and MDA5 (DKO) with PICV. We found that these $\mathrm{KO}$ mice experienced significant body weight loss and had significantly higher levels of infectious virus particles early in the infection that were cleared later during the course of the infection. These KO mice also had significantly depressed levels of IFN-I responses, but rather unexpectedly, appear to show similar levels of ISG expression as the WT mice and a heightened level of adaptive immune responses later in the course of the infection. Additionally, when a mutant PICV virus (rPICV-NPmut) that is known to be unable to suppress IFN-I expression was used to infect the WT as well as the SKO and DKO mice, all mice were able to effectively control virus infection, which further emphasizing the important roles of innate and adaptive immune responses that include but are not necessarily limited to RIG-I and MDA5, in controlling PICV infection in mice.

\section{METHODS}

\section{Plasmids and Cells}

The pCAGGS mammalian expression vectors for generating PICV and PICV-NP-D380A (rPICV-NPmut) viruses have been described previously $(34,35)$. For expression and purification of the recombinant NP protein, the full-length NP gene was cloned into the pRSF-duet bacterial expression plasmid and contained a $\mathrm{N}$-terminal histidine tag for expression in and purification from bacterial cells. Baby hamster kidney cells (BHK21) and African green-monkey kidney cells (Vero) were cultured in minimal essential media (MEM) supplemented with $10 \%$ heat-inactivated fetal bovine serum (FBS) and $50 \mu \mathrm{g} / \mathrm{mL}$ penicillin-streptomycin. BSRT7-5 cells, which were obtained from K.-K. Conzelmann (Ludwig-Maximilians-Universität, Germany), were cultured in minimal essential media (MEM) supplemented with $10 \%$ heat-inactivated fetal bovine serum (FBS) and $50 \mu \mathrm{g} / \mathrm{mL}$ penicillin-streptomycin.

\section{Virus Production and Sequencing}

The methods for generating rPICV and rPICV-NPmut viruses have been described previously $(35,36)$. Supernatants containing these recombinant rPICVs were collected at 48 and 72 hours post PICV reverse genetics plasmids transfection. The rPICVs were amplified once in BHK21 cells, and the virus titers were determined by plaque assay. Viral RNA was extracted from viral stocks (or infectious viral plaques) using the QIAamp Viral RNA kit (Qiagen, USA), amplified by RT-PCR and sequenced to confirm the identity of the virus stocks. The lack of WT rPICV 
in the rPICV-NPmut virus stock was confirmed using a nested $\mathrm{PCR}$ reaction with internal primers specific to the $\mathrm{WT}$ and mutant sequences as described previously (35). Primer sequences used for viral sequencing reactions are provided in Table $\mathbf{1}$.

\section{Mouse Lines and Handling}

We used the approved protocol of the Institutional Animal Care and Use Committee (IACUC) at the University of Minnesota to conduct animal studies. MDA5 KO and MDA5/RIG-I DKO C57BL/6J mice were kind gifts of Dr. Michael Gale (University of Washington), and their generation has been described previously (37). WT C57BL/6J mice were obtained from the Jackson Laboratory (USA). Age-matched male and female mice between 6 and 20 weeks of age were infected with rPICVs via the intra-peritoneal (IP) route, and body weight was monitored daily following the infection. Blood was taken via the facial veins at various time points following the infection and used for analysis of IFN $\beta$ and ISG expressions, viremia, CD8+ T cells and antibody titers. At 3-, 6-, 9- and 15-days post infection, mice were euthanized, and organs and serum were collected for viral titer determination by plaque assay and for RNA extraction.

\section{Plaque Assay}

Organs were prepared for plaque assay after tissue homogenization. Vero cells were seeded into 6-well plates at $60-70 \%$ confluence $\left(4 \times 10^{5}\right.$ cells/well). In the following day, cells were infected with $500 \mu \mathrm{L}$ of serially diluted rPICV stocks or of the organ homogenate in phosphate-buffered saline (PBS) for 1 hour at $37^{\circ} \mathrm{C}$. After washing once with PBS, cells were incubated in MEM supplemented with $0.5 \%$ agar and cultured for an additional 4 days at $37^{\circ} \mathrm{C}$ and $5.0 \% \mathrm{CO}_{2}$. Plaques were stained overnight with a diluted neutral red solution $(0.01 \%)$ in $0.5 \%$ agar-MEM medium.

\section{Quantification of IFN $\beta$ Expression by ELISA}

The IFN $\beta$ level in serum of the rPICV-infected mice was quantified using a mouse IFN $\beta$ enzyme-linked immunosorbent assay (ELISA) kit (LEGEND $\mathrm{MAX}^{\mathrm{TM}}$ Mouse IFN $\beta$ ELISA Kit

TABLE 1 | Primer sequences used in PCR and RT-qPCR reactions.

\begin{tabular}{ll}
\hline Primer Name & \multicolumn{1}{c}{ Sequence } \\
\hline $\begin{array}{l}\text { PICV NP PCR for mutation detection } \\
\text { and sequencing primer- } F\end{array}$ & GGCATCAGCCAAGTCCTTA \\
PICV NP PCR for mutation detection- R & TGTCTCAGCCTGGTGTTATGG \\
Mouse ISG54 F & ATGAAGACGGTGCTGAATACTAGTGA \\
Mouse ISG54 R & TGGTGAGGGCTTCTITTCC \\
Mouse ISG56 F & TGGCCGTTTCCTACAGIT \\
Mouse ISG56 R & TCCTCCAAGCAAAGGACTTC \\
Mouse ISG15 F & CTGAAGAAGCAGATTGCCCAGAAG \\
Mouse ISG15 R & CGCTGCAGTTCTGTACCACTAGC \\
Mouse IFN $\gamma \mathrm{F}$ & GAGGTCAACAACCCACAGGTC \\
Mouse IFN $\gamma$ R & CGAATCAGCAGCGACTCCT \\
Mouse IFN $F$ & AGCTGCAGGCCTTCAAAAAG \\
Mouse IFN $x$ R & TGGGAGTGAATGTGGCTCAG \\
Mouse B-actin F & GGTCATCACTATTGGCAACG \\
Mouse B-actin R & ACGGATGTCAACGTCACACT
\end{tabular}

with Pre-coated Plates) (Biolegend, USA) following the manufacturer's instructions.

\section{RNA Extraction and RT-qPCR}

Total RNA was extracted from spleens collected at 3- and 6-days post rPICV infection using TRIzol reagent (Life Technologies, USA). Single-stranded cDNAs were generated by the use of an oligo(dT) primer and MMLV reverse transcriptase (Promega, USA) following the manufacturer's protocol. Quantitative RTqPCR was conducted using specific primers designed to detect certain mouse genes of interest (Table 1) by using the IQ SYBR Green supermix (Bio-Rad, USA) in a Bio-Rad CFX quantitative PCR machine (Bio-Rad, USA) at $95^{\circ} \mathrm{C}$ for $3 \mathrm{~min}$, followed by 40 cycles of DNA amplification at $95^{\circ} \mathrm{C}$ for $10 \mathrm{~s}$ and $60^{\circ} \mathrm{C}$ for $30 \mathrm{~s}$. The data for each of the gene expressions were first normalized to the housekeeping gene $\beta$-actin and then compared to the level of the WT rPICV-infected mice using the $2^{-\Delta \Delta \mathrm{CT}}$ method.

\section{PICV NP Tetramer Analysis}

Blood was collected from mice in lithium-heparin tubes for peripheral blood monocyte (PBMC) preparation. PBMCs were isolated from whole blood using lymphoprep separation technique (Fisher, USA) followed by lysis of any remaining red-blood cells using the RBC lysis buffer (Thermo-Fisher, USA). Cells were blocked with anti-mouse CD16/32 antibody (BD Biosciences, USA), and incubated with PE-labeled PICV NP $(48-45) / \mathrm{H} 2 \mathrm{~K}$ (b) MHC-I tetramer, provided by the NIH tetramer core facility at Emory University, together with CD8a-PerCPCy5.5 (eBioscience, USA), CD3-APC (Biolegend, USA), Zombie violet stain (Biolegend, USA), for $30 \mathrm{~min}$ on ice in the dark. After washing, cells were analyzed by flow cytometry on a BD FACSCelesta Cell Analyzer machine (BD Biosciences, USA). Data was analyzed with the FlowJo software (FlowJo LLC, USA).

\section{Anti-PICV NP IgG ELISA}

pRSF-duet-P18NP was transformed into BL21 competent bacterial cells. After overnight IPTG induction $(0.1 \mathrm{mM})$ at $16^{\circ} \mathrm{C}$, the cells were lysed and the NP protein was purified on a HisTrap immobilized metal affinity chromatography (IMAC) column (Cytiva, USA). The Nunc MaxiSorp plates (Thermofisher, USA) were coated with $200 \mathrm{ng}$ of recombinant NP proteins overnight at $4^{\circ} \mathrm{C}$ in coating buffer $(50 \mathrm{mM}$ CarbonateBicarbonate buffer, $\mathrm{pH}$ 9.5). Plates were washed once with wash buffer (PBS $+0.2 \%$ Tween 20) and blocked for $2 \mathrm{~h}$ at ambient temperature with blocking buffer (PBS $+4.0 \%$ dry milk) followed by three more washes. Serum samples were serially diluted in diluting buffer (PBS $+0.05 \%$ Tween 20 ), added to plates, and incubated for $1 \mathrm{~h}$. Following three washes, samples were incubated with goat anti-guinea pig IgG HRP secondary antibody (Sigma, USA) at 1:10,000 dilution for $1 \mathrm{~h}$. Following four washes, samples were incubated with the TMB substrate (Thermo-fisher, USA) for $15 \mathrm{~min}$ in the dark, then a stop solution $\left(0.16 \mathrm{M} \mathrm{H}_{2} \mathrm{SO}_{4}\right)$ was added and optical density (OD) values were read at $450 \mathrm{~nm}$ using a Biotek Synergy 2 plate reader (Biotek, USA). The IgG antibody endpoint titer was defined as the highest dilution giving $\mathrm{OD}_{450}$ above the cutoff value, which 
was determined by average plus four times standard deviation of secondary-antibody-only controls.

\section{Statistical Analysis}

All statistical analyses were performed using GraphPad Prism (GraphPad Software, USA). The statistical significance of the differences in the mean values was analyzed using an unpaired, two-tailed Student's t test.

\section{RESULTS}

\section{MDA5 SKO and RIG-I/MDA5 DKO Mice Experience Significant Weight Loss During WT rPICV Infection}

To determine the importance of RIG-I and/or MDA5 against rPICV infection, body weight and morbidity data were collected daily for WT BL6, MDA5 KO and RIG-I/MDA5 DKO mice infected intra-peritoneally (IP) with $1.0 \times 10^{5} \mathrm{PFU}$ of the WT rPICV. While WT animals experienced no obvious signs of morbidity, $20 \%$ of the MDA5 SKO and almost 50\% of the RIG-I/MDA5 DKO mice showed significant levels of body weight loss of at least $10 \%$ after day 6 post infection, but ultimately began to recover body weight after 9 days post infection (Figure 1). Notably, the overall changes in body weight were significantly different for the infected SKO and DKO mice as compared to the WT mice, but the differences were not significant between the infected SKO and DKO mice. These data suggest that the RIG-I and MDA5 innate immune pathway acts as a key barrier against rPICV infection in mice.

\section{RIG-I and MDA5 Are Essential for Controlling Early WT rPICV Replication in Mice}

RIG-I and MDA5 are most canonically known as early cellular protein sensors of viral infection and are critical for controlling viral replication (21). To establish a timeline of $\mathrm{rPICV}$ replication in mice and to examine the possible connectivity between morbidity and the levels of viral replication, mice infected with WT rPICV were sacrificed at various timepoints post infection to determine infectious viral titers in the spleen, serum, and liver by plaque assay. As previously observed in rPICV-infected guinea pigs $(24,25,38)$, rPICV viral titer was highest in the spleen at day 3 post infection, which is thought to be due to the circulating monocytes trafficking into the draining lymph nodes being among the earliest targets of mammarenaviral infections (39, 40). Splenic virus titers were higher for the SKO and DKO mice as compared to the WT mice by several logs at day 3 post infection (Figure 2A) and were also significantly higher for both $\mathrm{KO}$ mouse lines in the liver (Figure $\mathbf{2 B}$ ) and for the DKO mice in the serum (Figure $2 \mathrm{C}$ ) at day 3 post infection. Notably, rPICV appeared to be cleared in the WT mice by day 6 and in the SKO and DKO mice by day 9 post infection, and while some virus titers were detected in a portion of the $\mathrm{KO}$ mouse lines in the spleen and liver at day 6, almost all $\mathrm{KO}$ mice had detectible viremia in the serum at day 6 (Figure 2C). Collectively, these data indicate that both RIG-I and MDA5 are essential for controlling early PICV replication in mice and that other cellular factors might be responsible for virus clearance later in the infection.

\section{WT rPICV-Infected MDA SKO and RIG-I/ MDA5 DKO Mice Express Interferon- Stimulated Genes (ISGs) Despite a Seemingly Lack of Early IFN-I Signaling via the RIG-I and MDA5 Pathway}

We decided to first confirm whether RIG-I and MDA5 KO prevented the onset of an early IFN-I response to WT rPICV infection by performing ELISA on the serum taken $24 \mathrm{~h}$ post infection when IFN $\beta$ is known to reach peak systemic levels (41, 42). As expected, the rPICV-infected WT mice had significantly higher levels of serum IFN $\beta$ than the rPICV-infected SKO and

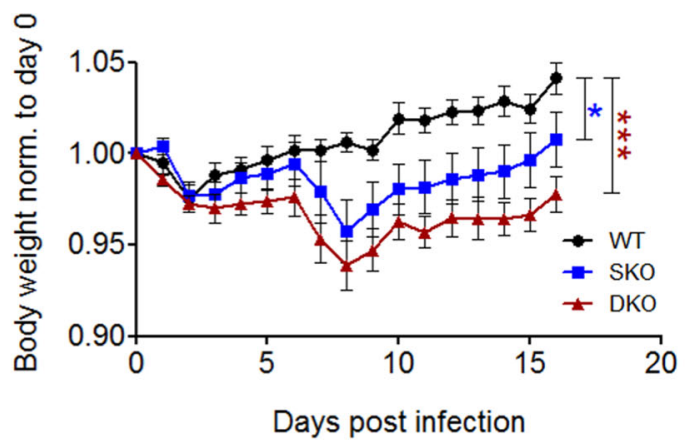

\begin{tabular}{llll}
\hline & WT & SKO & DKO \\
\hline $\begin{array}{l}\text { \# Animals with } \\
\text { >10\% body } \\
\text { weight loss }\end{array}$ & $0 / 18$ & $4 / 19$ & $8 / 19$ \\
$\begin{array}{l}\text { 95\% Confidence } \\
\text { interval }\end{array}$ & 0 & 3.48 & 4.22 \\
\hline
\end{tabular}

FIGURE 1 | MDA5 KO and RIG-I/MDA5 DKO mice experience weight loss during rPICV infection. Body weight was measured daily for WT ( $\mathrm{n}=18$ ), MDA5 SKO $(n=19)$ and DKO $(n=19)$ mice infected intraperitoneally $(I P)$ with $1 \times 10^{5}$ PFU of WT rPICV. The data are representative of 4 independent experiments. The significance values are reported as the level of statistical significance experienced each day between 2 groups after day 6 of infection when mice began to show weight loss. The number of animals in each group that experienced significant weight loss (defined as $>10 \%$ ) as well as the $95 \%$ confidence intervals for the proportion of animals that experienced significant body weight loss are reported in the table. ${ }^{\star} p<0.05,{ }^{\star \star} p<0.01,{ }^{\star \star \star} p<0.001$. 
A

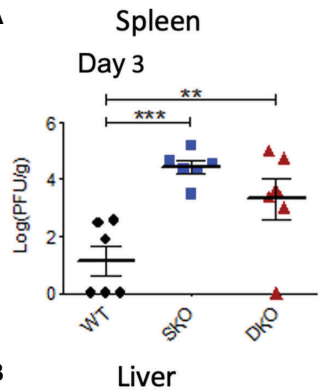

Day 3

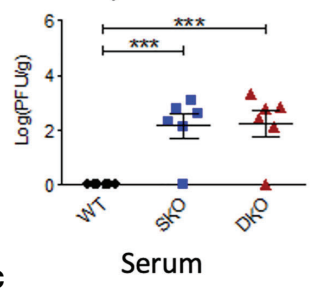

Day 3

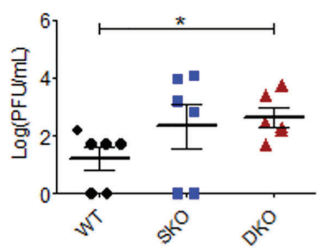

Day 6

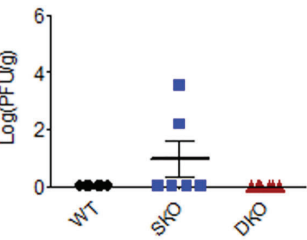

Day 6

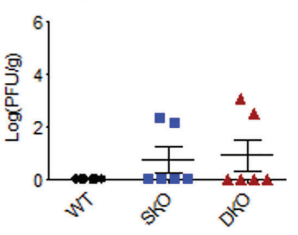

Day 6

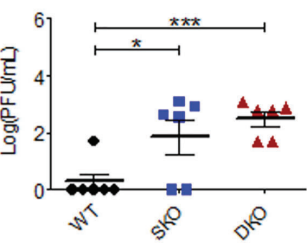

Day 9

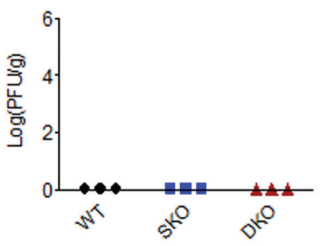

Day 9

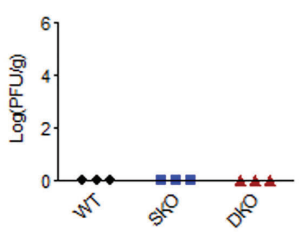

Day 9

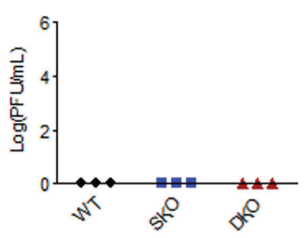

FIGURE 2 | RIG-I and MDA5 are essential for controlling early WT rPICV replication in mice. rPICV infectious viral titers were determined by plaque assay and are reported for spleen (A), liver (B), and serum (C) from the infected mice at 3-day intervals following the infection. The data are representative of 2 independent experiments. ${ }^{\star} p<0.05,{ }^{\star \star} p<0.01,{ }^{\star \star \star} p<0.001$.

DKO mice, indicating that these $\mathrm{KO}$ animals failed to launch a typical early and robust level of IFN-I expressions against rPICV infection (Figure 3A). Next, to test how the innate immune response progresses throughout the course of the WT rPICV infection, we quantified the gene expression levels of three well studied ISGs in splenic RNAs prepared at days 3 and 6 post infection by RT-qPCR. We measured the levels of gene expression of the ISG54, which is associated with cellular apoptosis (43), ISG56, which is known to be broadly inhibitive of cellular and viral replication (44), and ISG15, which induces proliferation and maturation of immune cells (45). RT-qPCR analysis showed no significant differences between rPICVinfected WT and SKO and DKO mice in ISG responses (Figures 3B, D), indicating that these two KO mouse lines were somehow able to express these particular ISGs by day 3 post viral infection despite the lack of early IFN-I signaling mediated by the RIG-I and MDA5 pathway. We also measured the levels of gene expression of the type II IFN (IFN $\gamma$ ) at days 3 and 6 post infection by RT-qPCR. There was also no significant difference in IFN $\gamma$ expression at day 3 , though there was a slightly significant increase in IFN $\gamma$ expression in the SKO mice at day 6 (Figure 3E), the significance of which is not known. Taken together, these data indicate that while RIG-I and MDA5 are key regulators of IFN-I expression early during rPICV infection, mice lacking these cellular proteins may still be able to express a certain type of ISG genes during rPICV infection.

\section{WT rPICV-Infected MDA5 SKO and RIG-I/ MDA5 DKO Mice Exhibited Heightened Levels of Adaptive Immune Responses to Virus Infection}

While not much is known about the specific roles of RIG-I and MDA5 in activating the adaptive immune responses to viral infection, IFN-I is well known to be critical for activating $\mathrm{T}$ and $\mathrm{B}$ cells to control virus infection $(20,46)$. Notably, effective adaptive immune responses [especially cellular immunity $(15,47)$ ] have been shown to be critical for clearing LASV infection and for the Lassa fever recovery in humans $(48,49)$. We therefore decided to explore the potential impacts of RIG-I and MDA5 on the adaptive immune responses to WT rPICV infection of mice. First, we obtained PBMCs from the WT rPICV-infected mice at weekly intervals following virus infection and stained them with a known PICV-NP MHCI tetramer together with CD8+ T cell markers (Figure 4A). We found that the SKO and DKO mice developed stronger PICV-specific CD8+ T cells than WT mice, with the greatest levels of differences at day 14 post infection when tetramer+ CD8+ T cells peaked for both of the $\mathrm{KO}$ mouse lines (Figure 4B). We next quantified the levels of 
A

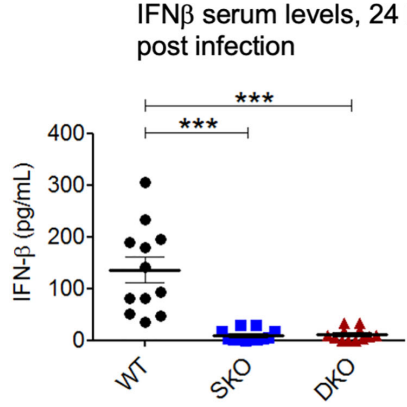

C

ISG56

Day 3

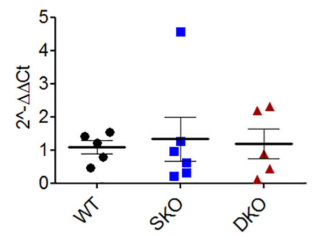

E

IFN $\gamma$

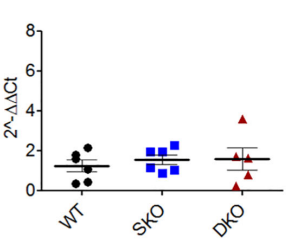

Day 6
B

ISG54
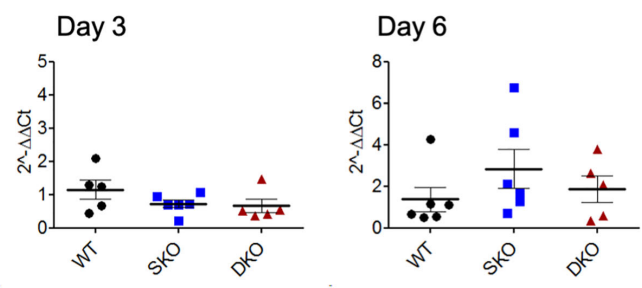

D

ISG15
Day 3

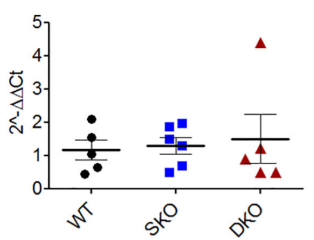

Day 6

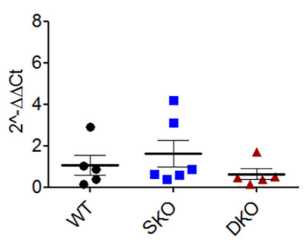

Day 3

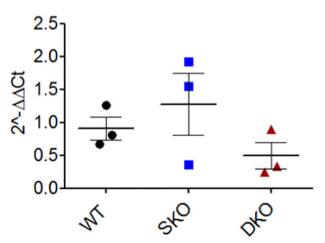

Day 6

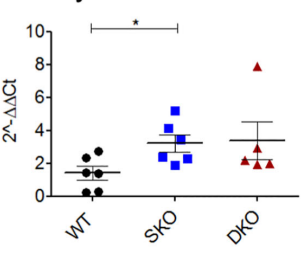

FIGURE 3 | MDA5 SKO and RIG-I/MDA5 DKO mice have decreased early IFN-I signaling but express ISGs later in the course of the infection. Serum was isolated from whole blood taken $24 \mathrm{~h}$ after WT rPICV infection, and IFN-B levels in the serum were determined by ELISA (A). Total RNAs were extracted from spleens at 3 days and 6 days post WT rPICV infection and were analyzed by RT-qPCR to determine the expression levels of the ISG54 (B), ISG56 (C), ISG15 (D) and IFN- $\gamma$ (E). Data are representative of 2 independent experiments. ${ }^{\star} p<0.05,{ }^{\star \star} p<0.01,{ }^{\star \star \star} \mathrm{p}<0.001$.

anti-PICV antibody response at days 7-21 post infection by ELISA and found that the anti-PICV antibody titers in both of the KO mouse lines were 1-2 log higher than those in the rPICV-infected WT mice at days 14 and 21 (Figure 4C). Taken altogether, these data indicate that despite the lack of early IFN-expressions mediated by RIG-I and MDA5, mice lacking these innate immune regulatory gene expressions are still able to mount effective adaptive immune responses against $\mathrm{rPICV}$ infection in mice.

\section{Absence of RIG-I and MDA5 Does Not Necessarily Abolish Anti-Viral Responses to Viral PAMP dsRNAs}

One of the ways that mammaviruses evade the innate immune recognition upon virus infection is through the viral nucleoprotein (NP) exoribonuclease (ExoN) activity that can degrade PAMP dsRNAs (50-52). rPICV containing an ExoN- deficient NP has been shown to replicate at significantly higher levels in RIG-I KO mouse embryonic fibroblasts (MEFs) than in WT cells and can induce a strong level of IFN-I response in the WT cells (53).

To determine the potential role of NP's ExoN in rPICVinfected mice, we infected WT, MDA5 SKO, and RIG-I/MDA5 DKO mice with the rPICV-NPmut virus that contains a D380A mutation in NP gene. This D380A mutation has previously been shown to abolish the NP's ExoN enzymatic activity and therefore can induce strong IFN-I responses in cell culture as well as in the rPICV-NPmut virus-infected guinea pigs (35). In the current study, none of the rPICV-NPmut-infected mice lose significant body weights during the course of the infection as compared to the rPICV-NPmut-infected WT mice (Figure 5A) with only two out of 21 SKO and DKO mice produced detectable levels of virus replication by plaque assay (Figure 5B). It is noteworthy that 
A
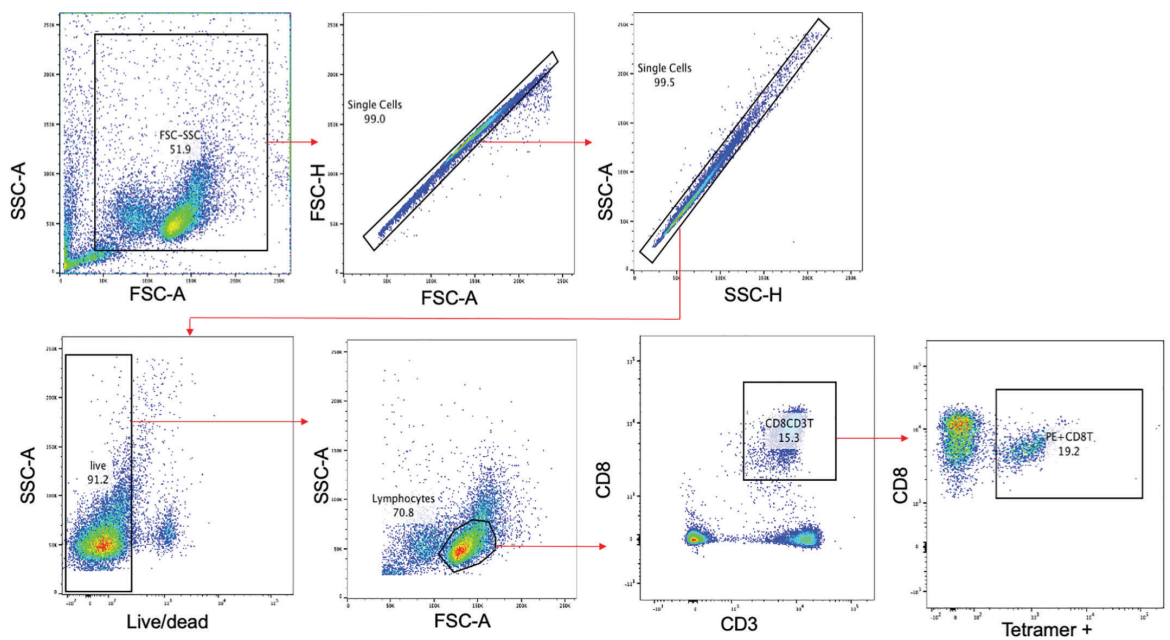

B

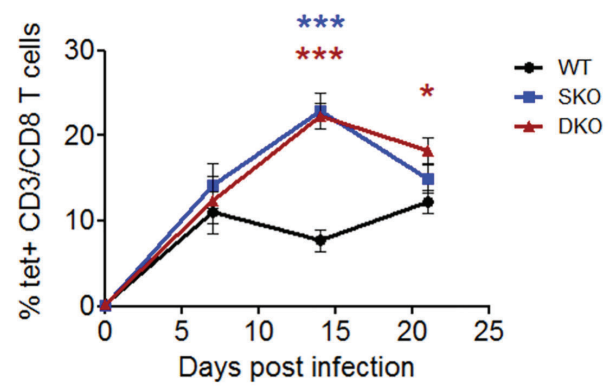

C

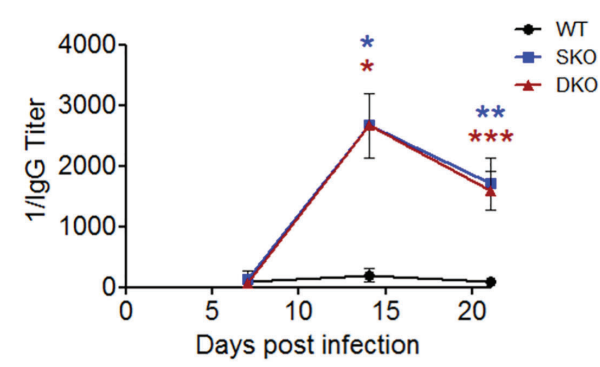

FIGURE 4 | MDA5 SKO and RIG-I/MDA5 DKO increases adaptive immune responses to rPICV infection. (A) PBMCs were isolated from whole blood taken at 7 days, 14 days and 24 days post WT rPICV infection and were stained to analyze the levels of rPICV-specific CD8+ T cell responses. Cells were gated using the following gating strategy: whole cells (FSC-A/SSC-A), single cells (FSC-A/FSC-H, then SSC-H/SSC-A), live/dead (BV421/SSC-A), lymphocytes (FSC-A/SSC-A), CD8 T cells (APC-CD3/PerCP 5.5 CD8), P18 specific CD8 T cells (PE-tet/Percp 5.5 CD8). (B) The percentages (\%) of CD8+ T cells that were also tetramer positive were reported at 7 -day intervals during infection $(n=4)$. (C) Sera were isolated from whole blood taken at day 7,14 and 21 post $r P I C V$ infection, and anti-PICV NP antibody titers were determined by ELISA. ${ }^{*} \mathrm{p}<0.05,{ }^{* *} \mathrm{p}<0.01,{ }^{* * *} \mathrm{p}<0.001$.

sequencing of the viruses recovered from those two rPICVNPmut-infected SKO and DKO mice showed that all the viruses with the original NP D380A mutations have reverted to the WT sequence (Figure 5C), implicating the strong preference of the rPICV to maintain the NP's ExoN function in vivo, even in the absence of a functional RIG-I and MDA5 immune surveillance system. Furthermore, whereas the WT mice infected with the WT rPICV produced high levels of early IFN $\beta$ and ISGs at day 3 post infection that were diminished by day 6 post infection (Figures 5D-G), none of the rPICVNPmut-infected mice produced significant levels of these genes at both time points, except for a comparable level of ISG15 expression in the $\mathrm{KO}$ mouse lines at day 3 post infection as compared to that in the WT mice infected with the WT rPICV, the significance of which is not known.

Interestingly, rPICV-NPmut induced a delayed and transient $\mathrm{T}$ cell response in a subset of mice from all three genetic backgrounds. Specifically, the PICV-specific T-cells were only being detected starting at 14 days in the rPICV-NPmut-infected mice as compared to 7 days in the WT rPICV-infected mice. Additionally, the PICV-specific T-cells in the rPICV-NPmutinfected mice diminished by day 20 post infection (Figure $\mathbf{5 H}$ ). Similarly, the anti-PICV antibody responses were significantly lower in the rPICV-NPmut-infected mice than in the WT rPICV-infected mice, with the strongest antibody responses observed in animals with the strongest $\mathrm{T}$ cell responses (Figure 5I). The differential levels of adaptive immune responses likely reflect the differences in the level of viral replication in vivo, as higher viral load generally induces stronger immune responses.

\section{DISCUSSION}

In this study, we have established the important roles of RIG-I and MDA5 as key innate-immune cellular factors for the natural protection of mice against PICV infection. This finding falls in line with previous studies of infection of RIG-I and MDA5 KO mice with 


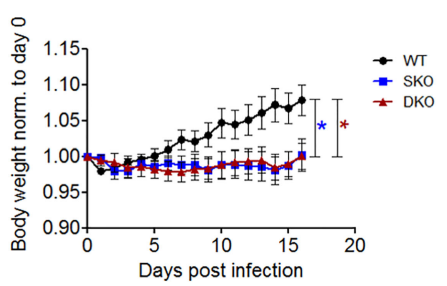

C

\begin{tabular}{ll}
\hline Genotype & \# WT revertant plaques \\
SKO & $4 / 4$ \\
DKO & $5 / 5$ \\
\hline
\end{tabular}

E

ISG54

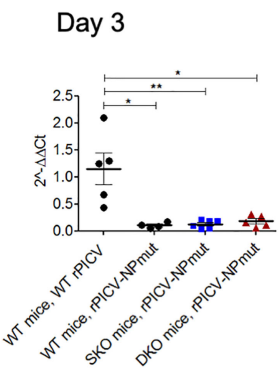

Day 6

G ISG15

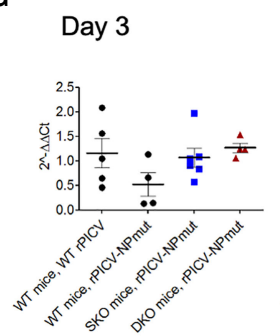

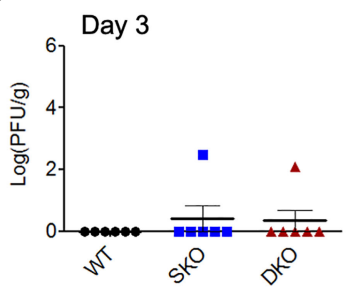

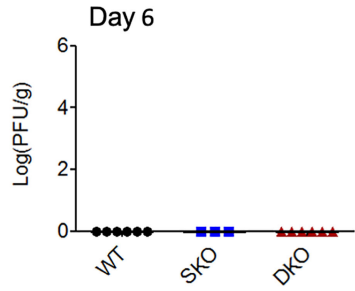

D

IFN $\beta$ serum levels, 24 hours post infection

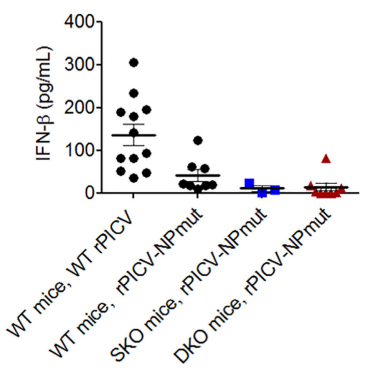

F

ISG56

Day 3

Day 6
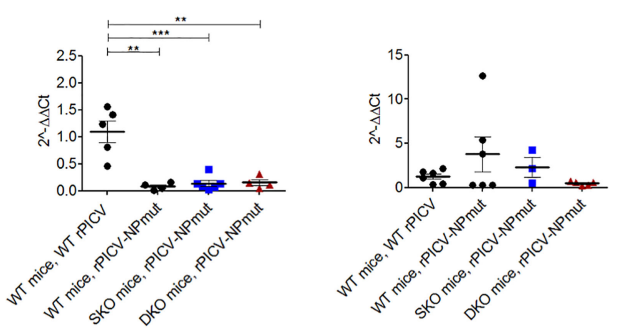

H

I
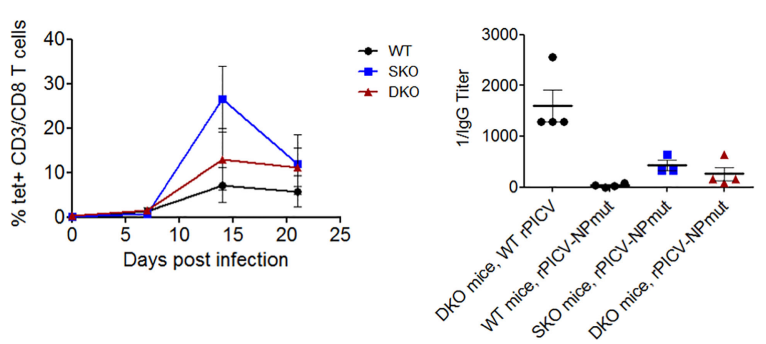

FIGURE 5 | WT and KO mice robustly control rPICV-NPmut infection. (A) Body weight was measured daily for WT ( $\mathrm{n}=10)$, MDA5 SKO $(\mathrm{n}=11)$ and RIG-1/MDA5 DKO $(n=11)$ mice infected intraperitoneally $(I P)$ with $1 \times 10^{5}$ PFU of rPICV-NPmut virus. Statistical significance was observed in comparing WT to SKO and DKO between days 12 and 16 post infection. (B) rPICV-NPmut infectious viral titers were determined by plaque assay and are reported for spleen at days 3 and 6 following rPICV-NPmut infection. (C) Total RNAs were extracted from individual viral plaque from some of the virus-infected spleens and were sequenced to confirm the identity of the intended Alanine substitution at residue 380 of the PICV NP gene of the rPICV-NPmut and of the NP WT-revertant with the Asparagine at this position. (D) Sera were isolated from whole blood taken $24 \mathrm{~h}$ after rPICV-NPmut infection, and the IFN-B expression levels in the sera were determined by ELISA and compared to the IFN-B expression levels seen with the WT mice infected with the WT rPICV. (E-G) Total RNAs were extracted from spleens taken 3- and 6-days post rPICV-NPmut infection and were analyzed by RT-qPCR to determine the expression levels of the ISG54, ISG56 and ISG15. (H) The percentages (\%) of CD8+ T cells that were also tetramer positive were reported at 7-day intervals $(n=4)$. (I) Sera were isolated from whole blood taken at day 21 post rPICV-NPmut, and the levels of anti-PICV NP antibody were determined by ELISA. ${ }^{\star} p<0.05,{ }^{* \star} p<0.01,{ }^{\star \star *} p<0.001$.

other RNA viruses. For example, certain strains of the lymphocytic choriomeningitis virus (LCMV), an OW mammarenavirus, had been used in a variety of model organisms due to its ability to induce symptoms in immunocompetent animals that range from a lethal disease in rhesus monkeys (54) to a chronic infection in mice that is considered a good model to study $\mathrm{T}$ cell responses to infection and $\mathrm{T}$ cell exhaustion $(55,56)$. LCMV-infected MDA5 SKO mice demonstrated significantly lower IFN-I signaling than in the virus- 
infected WT mice $(41,42)$. They also had significantly higher splenic viral titers and delayed $\mathrm{T}$ cell responses up to 3 weeks as compared to the virus-infected WT animals (42). Similar findings were found using West Nile virus infection in MDA5 SKO mice (57) and in RIG-I SKO, MDA5 SKO and DKO mice (37) with notable differences in boosted IFN-I signaling in the MDA5 SKO mice (57). It was noted that RIG-I SKO mice infected with the influenza virus PR8 had significantly longer viral clearance times and lower numbers of activated T cells than the infected WT mice (58). Finally, it was found that mice treated with RIG-I-activating ligand prior to infection by SARS-CoV-2 had increased survivability, decreased viral titers and increased antibody responses as compared to the virus-infected WT mice, as described in a recent preprint (59). Taken together, these findings indicate that RIG-I and MDA5 consistently provide antiviral protection in mice by inducing early IFN-I expression to inhibit virus replication, as shown in our current study (Figures 2, 3).

It is also notable that we could recover WT revertant PICVs from two of the mice infected with the rPICV-NPmut (Figure 5C). WT PICV revertants have also previously been recovered in the serum of guinea pigs infected with the same rPICV-NPmut (35), indicating that there is a strong selective pressure in both mice and guinea pigs to maintain a functional PICV NP ExoN for optimal virus replication in these animals. This is consistent with the fact that ExoN-deficient rPICV experiences significantly attenuated growth kinetics as compared to the WT rPICV in cell culture $(35,53)$. It is also important to note that the WT revertant PICVs were derived in the MDA5 SKO and RIG-I/MDA5 DKO mice (Figure 5C), which strongly suggests either an active immune pressure conferred by cellular factors besides RIG-I and MDA5 or the propensity of the virus to maintain an active NP ExoN function for optimal virus replication.

It is well documented that anti-viral activity must also rely on other innate immune sensors, such as the Toll-like receptors (TLRs) (60), protein kinase R (PKR) (61) and Laboratory of Genetics and Physiology protein 2 (LGP2) (62), all of which can also recognize either PAMP RNAs or various other aspects of the pathogens upon virus infection. In the context of mammarenaviral infection, activation of these innate immune receptors has been documented, in particular, with the NW Junin virus (JUNV) infection, in which a strong and sustained inflammatory response is likely associated with the development of severe disease $(6,63,64)$. The TLR2/6 complex has been shown to be critical for antiviral activity against JUNV infection $(65,66)$. Likewise, PKR has been found to be activated upon JUNV infection in cell culture, but it did not have a significant impact on virus replication (67). While it is possible that the innate immune responses initiated by PICV infection may be similar to JUNV and other NW mammarenavirus infections, further studies are needed to fully understand the immune responses to PICV infection in comparison to other pathogenic vs. non-pathogenic mammarenaviruses of both $\mathrm{NW}$ and $\mathrm{OW}$ groups, and in different experimental systems.

It is also possible that expression of other members of the interferon family contributed to the expression of ISGs in the KO mice. The IFN-I family consists of several members, the most predominant being IFN $\alpha$ and IFN $\beta$ but also including others, such as IFNא, IFN $\omega$ and IFN $\tau$ (68). It is noteworthy that ISG expressions can also be stimulated by the type II (IFN $\gamma$ ) and type III (IFN $\Lambda$ ) interferons (69-71). Some studies have characterized the expression of type II interferons during mammarenaviral infection (72-78). In the current study, we found no significant difference in IFN $\gamma$ expression in day 3 and a slightly significant increase of IFN $\gamma$ expression in the SKO mice at day 6. This may be correlated with the strengthened CD8 T cell responses in these mice given the well characterized role of IFN $\gamma$ in initiating $\mathrm{T}$ cell growth (79) and its subsequent expression by activated T cells (70). However, we did not find any quantifiable expression of IFN $\Lambda$ in the spleen of infected mice (data not shown). This is consistent with previous finding of IFN $\Lambda$ being most highly expressed in mucosal organs, such as the lungs, but at very low levels in the spleen (80). Unfortunately, no lung samples were preserved for the IFN $\Lambda$ analysis in our study. Regardless, we believe that type II (but not likely type III) interferons may play an important role in mediating splenic ISG expressions in the KO mice.

We also noted a higher level of adaptive immune responses to rPICV infection in the SKO and DKO mice than in the WT mice (Figures 4B, C). It is possible that a relatively small level of IFN-I expressions (Figure 3A) in these genetically altered mice in response to an active virus replication (Figure 2) can still trigger robust levels of ISG expressions (Figures 3B-D) and cellular immunity (Figure 4). A stronger level of adaptive immune responses in the SKO and DKO mice at the later timepoints during the infection strongly indicates that both humoral and adaptive immune responses might be necessary to fully control the infection in these mice. This data falls in line with early studies showing potent $\mathrm{T}$ and NK responses in PICV infected mice $(81,82)$ that were critical for clearing viral infection (83). Adaptive immunity to PICV infection is also relevant considering that cellular immunity have been shown to be essential for clearing LASV infection $(15,47)$ and in preventing the development of serious disease $(48,49)$.

Apart from the data showing that more DKO mice experiencing significant weight loss than the SKO mice during WT rPICV infection, we did not observe any other significant differences between these genetically altered mouse lines. However, previous studies have suggested that RIG-I is more important in controlling replication of many known negativesense RNA viruses, including mammarenaviruses $(53,84,85)$, whereas MDA5 is important in controlling many positive-sense RNA virus infections $(21,23)$. This is likely due to the differences in the RNA ligands that these viruses produce during the course of the infection, with RIG-I mostly responding to short, doublestranded phosphorylated RNAs, and MDA5 responding to long double-stranded RNAs (21). However, in West Nile virus infection (86), RIG-I and MDA5 appear to have an additive anti-viral effect, whereas either of these two cellular proteins can provide protection against Dengue virus infection (87). Such complexities are likely driven by either the differing species of the PAMP RNAs generated during virus infections or other aspects 
of the viruses (e.g., viral lipids or proteins) that can also trigger IFN expressions via TLR activations, for instance.

In summary, we have demonstrated in the current study that RIG-I and MDA5 are important in providing protection against rPICV infection in mice by controlling the early stages of viral replication through IFN-I activation. Our data also suggest that RIG-I and MDA5 might not be the only cellular factors responding to $\mathrm{rPICV}$ infection but that activation of adaptive immunity may also be necessary to effectively clear the infection. Further studies are needed to understand the full spectrum of immune sensors and responses to PICV and other mammarenavirus infections.

\section{DATA AVAILABILITY STATEMENT}

The original contributions presented in the study are included in the article/supplementary material. Further inquiries can be directed to the corresponding author.

\section{ETHICS STATEMENT}

The animal study was reviewed and approved by University of Minnesota Institutional Animal Care and Use Committee.

\section{REFERENCES}

1. Charrel RN, de Lamballerie X, Emonet S. Phylogeny of the Genus Arenavirus. Curr Opin Microbiol (2008) 11:362-8. doi: 10.1016/j.mib.2008.06.001

2. Günther S, Lenz O. Lassa Virus. Crit Rev Clin Lab Sci (2004) 41:339-90. doi: 10.1080/10408360490497456

3. Damonte EB, Coto CE. Treatment of Arenavirus Infections: From Basic Studies to the Challenge of Antiviral Therapy. Adv Virus Res (2002) 58:12555. doi: 10.1016/S0065-3527(02)58004-0

4. Maiztegui J, Fernandez N, De Damilano A. Efficiacy of Immune Plasma in Treatment of Argentine Haemorrhagic Fever and Association Between Treatment and a Late Neurological Syndrome. Lancet (1979) 314:1216-7. doi: 10.1016/S0140-6736(79)92335-3

5. Koma T, Huang C, Kolokoltsova OA, Brasier AR, Paessler S. Innate Immune Response to Arenaviral Infection: A Focus on the Highly Pathogenic New World Hemorrhagic Arenaviruses. J Mol Biol (2013) 425:4893-903. doi: 10.1016/j.jmb.2013.09.028

6. Brisse ME, Ly H. Hemorrhagic Fever-Causing Arenaviruses: Lethal Pathogens and Potent Immune Suppressors. Front Immunol (2019) 10:372. doi: 10.3389/ fimmu.2019.00372

7. Prioritizing Diseases for Research and Development in Emergency Contexts. Available at: https://www.who.int/activities/prioritizing-diseases-forresearch-and-development-in-emergency-contexts (Accessed August 8, 2021).

8. Pfau CJ. Arenaviruses. In: Medical Microbiology. Galveston: University of Texas Medical Branch (1996). p. 501-41. Available at: https://www.ncbi.nlm. nih.gov/books/NBK8193/.

9. Lecompte E, Fichet-Calvet E, Daffis S, Koulémou K, Sylla O, Kourouma F, et al. Mastomys Natalensis and Lassa Fever, West Africa. Emerg Infect Dis (2006) 12:1971. doi: 10.3201/EID1212.060812

10. Mariën J, Sluydts V, Borremans B, Gryseels S, Vanden Broecke B, Sabuni CA, et al. Arenavirus Infection Correlates With Lower Survival of Its Natural Rodent Host in a Long-Term Capture-Mark-Recapture Study. Parasites Vectors (2018) 11:90. doi: 10.1186/s13071-018-2674-2

11. Mariën J, Borremans B, Gryseels S, Soropogui B, De Bruyn L, Bongo GN, et al. No Measurable Adverse Effects of Lassa, Morogoro and Gairo Arenaviruses

\section{AUTHOR CONTRIBUTIONS}

The authors confirm contribution to the paper as follows. Study conception and design: MB, HL, and YL. Data collection: MB, $\mathrm{QH}, \mathrm{MR}$, and DD. Analysis and interpretation of results: $\mathrm{MB}$, HL, and YL. Draft manuscript preparation: MB, HL, and YL. All authors reviewed the results and approved the final version of the manuscript.

\section{FUNDING}

This work was supported in part by the NIH NIAID grant R01 AI131586 to HL and YL and by a predoctoral NIH fellowship T32 DA007097 and the University of Minnesota Doctoral Dissertation Fellowship (DDF) to MB.

\section{ACKNOWLEDGMENTS}

The authors would like to thank M. Dileepan, K. Snyder, R. Hullsiek, and H. Kumar at the University of Minnesota for their technical assistance. We acknowledge the NIH Tetramer Core Facility (contract HHSN272201300006C) for provision of PICV NP(38-45) MHC-I tetramer.

on Their Rodent Reservoir Host in Natural Conditions Joachim. Parasites Vectors (2017) 10:210. doi: 10.1186/s13071-017-2146-0

12. Mandl JNN, Ahmed R, Barreiro LBB, Daszak P, Epstein JHH, Virgin HWW, et al. Reservoir Host Immune Responses to Emerging Zoonotic Viruses. Cell (2015) 160:20-35. doi: 10.1016/j.cell.2014.12.003

13. Raabe VN, Kann G, Ribner BS, Morales A, Varkey JB, Mehta AK, et al. Favipiravir and Ribavirin Treatment of Epidemiologically Linked Cases of Lassa Fever. Clin Infect Dis (2017) 65:855-9. doi: 10.1093/cid/cix406. Favipiravir

14. Johnson KM, McCormick JB, Webb PA, Smith ES, Elliott LH, King IJ. Clinical Virology of Lassa Fever in Hospitalized Patients. J Infect Dis (1987) 155:45664. doi: 10.1093/infdis/155.3.456

15. Baize S, Marianneau P, Loth P, Reynard S, Journeaux A, Chevallier M, et al. Early and Strong Immune Responses Are Associated With Control of Viral Replication and Recovery in Lassa Virus-Infected Cynomolgus Monkeys. J Virol (2009) 83:5890-903. doi: 10.1128/JVI.01948-08

16. McLay L, Liang Y, Ly H. Comparative Analysis of Disease Pathogenesis and Molecular Mechanisms of New World and Old World Arenavirus Infections. J Gen Virol (2014) 95:1-15. doi: 10.1099/vir.0.057000-0

17. Murphy HL, Ly H. Pathogenicity and Virulence Mechanisms of Lassa Virus and Its Animal Modeling, Diagnostic, Prophylactic, and Therapeutic Developments. Virulence (2021). doi: 10.1080/21505594.2021.2000290

18. Ng CT, Sullivan BM, Teijaro JR, Lee AM, Welch M, Rice S, et al. Blockade of Interferon Beta, But Not Interferon Alpha, Signaling Controls Persistent Viral Infection. Cell Host Microbe (2015) 17:653-61. doi: 10.1016/j.chom.2015.04.005

19. Wittling MC, Cahalan SR, Levenson EA, Rabin RL. Shared and Unique Features of Human Interferon-Beta and Interferon-Alpha Subtypes. Front Immunol (2021) 11:605673. doi: 10.3389/fimmu.2020.605673

20. McNab F, Mayer-Barber K, Sher A, Wack A, O'Garra A. Type I Interferons in Infectious Disease. Nat Rev Immunol (2015) 15:87-103. doi: 10.1038/nri3787

21. Brisse M, Ly H. Comparative Structure and Function Analysis of the RIG-ILike Receptors: RIG-I and MDA5. Front Immunol (2019) 10:1586. doi: 10.3389/FIMMU.2019.01586

22. Liu GQ, Gack MU. Distinct and Orchestrated Functions of RNA Sensors in Innate Immunity. Immunity (2020) 53:26-42. doi: 10.1016/j.immuni. 2020.03.017 
23. Loo Y-M, Fornek J, Crochet N, Bajwa G, Perwitasari O, Martinez-Sobrido L, et al. Distinct RIG-I and MDA5 Signaling by RNA Viruses in Innate Immunity. J Virol (2008) 82:335-45. doi: 10.1128/JVI.01080-07

24. Jahrling PB, Hesse RA, Rhoderick JB, Elwell MA, Moe JB. Pathogenesis of a Pichinde Virus Strain Adapted to Produce Lethal Infections in Guinea Pigs. Infect Immun (1981) 32:872-80. doi: 10.1128/iai.32.2.872-880.1981

25. Lan S, Shieh W-J, Huang Q, Zaki SR, Liang Y, Ly H. Virulent Infection of Outbred Hartley Guinea Pigs With Recombinant Pichinde Virus as a Surrogate Small Animal Model for Human Lassa Fever. Virulence (2020) 11 (1):1131-41. doi: 10.1080/21505594.2020.1809328

26. Rojek JM, Sanchez AB, Nguyen NT, de la Torre J-C, Kunz S. Different Mechanisms of Cell Entry by Human-Pathogenic Old World and New World Arenaviruses. J Virol (2008) 82:7677-87. doi: 10.1128/JVI.00560-08

27. Singh MK, Fuller-Pace FV, Buchmeier MJ, Southern PJ. Analysis of the Genomic L Rna Segment From Lymphocytic Choriomeningitis Virus. Virology (1987) 161:448-56. doi: 10.1016/0042-6822(87)90138-3

28. Hastie KM, Kimberlin CR, Zandonatti MA, MacRae IJ, Saphire EO. Structure of the Lassa Virus Nucleoprotein Reveals a dsRNA-Specific 3' to 5' Exonuclease Activity Essential for Immune Suppression. Proc Natl Acad Sci USA (2011) 108:2396-401. doi: 10.1073/pnas.1016404108

29. Perez M, Craven RC. Torre JC De La. The Small RING Finger Protein Z Drives Arenavirus Budding: Implications for Antiviral Strategies. Proc Natl Acad Sci USA (2003) 100:12978-83. doi: 10.1073/pnas.2133782100

30. Dhanwani R, Huang Q, Lan S, Zhou Y, Shao J, Liang Y, et al. Establishment of Bi-Segmented and Tri-Segmented Reverse Genetics Systems to Generate Recombinant Pichinde Viruses. Methods Mol Biol (2018) 1604:247-53. doi: 10.1007/978-1-4939-6981-4_19 PMCID: PMC6367673 NIHMSID: NIHMS1006106 PMID: 28986840

31. Polyak SJ, Rawls WE, Harnish DG. Characterization of Pichinde Virus Infection of Cells of the Monocytic Lineage. J Virol (1991) 65:3575-82. doi: $10.022-538 X / 91 / 073575-08 \$ 02.00 / 0$

32. Clark T, Gresser I, Pfau C, Moss J, Woodrow D. Antibody to Mouse Alpha/ Beta Interferon Abrogates Pichinde Virus-Induced Liver Lesions in Suckling Mice. J Virol (1986) 59:728-30. doi: 10.1128/jvi.59.3.728-730.1986

33. Kenney LL, Carter EP, Gil A, Selin LK. T Cells in the Brain Enhance Neonatal Mortality During Peripheral LCMV Infection. PloS Pathog (2021) 17: e1009066. doi: 10.1371/journal.ppat.1009066

34. Lan S, McLay L, Aronson J, Ly H, Liang Y. Genome Comparison of Virulent and Avirulent Strains of the Arenavirus Pichinde. Arch Virol (2008) 153:1241. doi: 10.1007/S00705-008-0101-2

35. Huang Q, Shao J, Lan S, Zhou Y, Xing J, Dong C, et al. In Vitro and In Vivo Characterizations of Pichinde Viral Nucleoprotein Exoribonuclease Functions. J Virol (2015) 89:6595-607. doi: 10.1128/JVI.00009-15

36. Lan S, McLay Schelde L, Wang J, Kumar N, Ly H, Liang Y. Development of Infectious Clones for Virulent and Avirulent Pichinde Viruses: A Model Virus To Study Arenavirus-Induced Hemorrhagic Fevers. J Virol (2009) 83:6357-62. doi: 10.1128/JVI.00019-09

37. Errett JS, Suthar MS, McMillan A, Diamond MS, Gale M. The Essential, Nonredundant Roles of RIG-I and MDA5 in Detecting and Controlling West Nile Virus Infection. J Virol (2013) 87:11416-25. doi: 10.1128/JVI.01488-13

38. Connolly BM, Jenson AB, Peters CJ, Geyer SJ, Barth JF, McPherson RA. Pathogenesis of Pichinde Virus Infection in Strain 13 Guinea Pigs: An Immunocytochemical, Virologic, and Clinical Chemistry Study. Am J Trop Med Hygiene (1993) 49:10-24. doi: 10.4269/ajtmh.1993.49.10

39. González PH, Cossio PM, Arana R, Maiztegui JI, Laguens RP. Lymphatic Tissue in Argentine Hemorrhagic Fever. Pathologic Features. Arch Pathol Lab Med (1980) 104:250-4.

40. Buchmeier MJ, de la Torre J-C, Peters CJ. Arenaviridae. In: Knipe DM, Holey P, editors. Fields Virology. Philadelphia, PA: Lippincott Williams \& Wilkins (2007). p. 1283-303.

41. Zhou S, Cerny AM, Zacharia A, Fitzgerald KA, Kurt-Jones EA, Finberg RW. Induction and Inhibition of Type I Interferon Responses by Distinct Components of Lymphocytic Choriomeningitis Virus. J Virol (2010) 84:9452-62. doi: 10.1128/JVI.00155-10

42. Wang Y, Swiecki M, Cella M, Alber G, Schreiber RD, Gilfillan S, et al. Timing and Magnitude of Type I Interferon Responses by Distinct Sensors Impact CD8 T Cell Exhaustion and Chronic Viral Infection. Cell Host Microbe (2012) 11:631-42. doi: 10.1016/j.chom.2012.05.003
43. Reich NC. A Death-Promoting Role for ISG54/IFIT2. J Interferon Cytokine Res (2013) 33:199. doi: 10.1089/JIR.2012.0159

44. Fensterl V, Sen GC. The ISG56/IFIT1 Gene Family. J Interferon Cytokine Res (2011) 31:71. doi: 10.1089/JIR.2010.0101

45. Perng Y-C, Lenschow DJ. ISG15 in Antiviral Immunity and Beyond. Nat Rev Microbiol 2018 16:7 (2018) 16:423-39. doi: 10.1038/s41579-018-0020-5

46. Crouse J, Kalinke U, Oxenius A. Regulation of Antiviral T Cell Responses by Type I Interferons. Nat Rev Immunol (2015) 15:231-42. doi: 10.1038/nri3806

47. Hallam HJ, Hallam S, Rodriguez SE, Barrett ADT, Beasley DWC, Chua A, et al. Baseline Mapping of Lassa Fever Virology, Epidemiology and Vaccine Research and Development. NPJ Vaccines (2018) 3:11. doi: 10.1038/s41541018-0049-5

48. ter Meulen J, Badusche M, Kuhnt K, Doetze A, Satoguina J, Marti T, et al. Characterization of Human CD4(+) T-Cell Clones Recognizing Conserved and Variable Epitopes of the Lassa Virus Nucleoprotein. J Virol (2000) 74:2186-92. doi: 10.1128/JVI.74.5.2186-2192.2000

49. Yun NE, Walker DH. Pathogenesis of Lassa Fever. Viruses (2012) 4:2031-48. doi: $10.3390 / \mathrm{v} 4102031$

50. Qi X, Lan S, Wang W, Schelde LM, Dong H, Wallat GD, et al. Cap Binding and Immune Evasion Revealed by Lassa Nucleoprotein Structure. Nature (2010) 468:779-83. doi: 10.1038/nature09605

51. Pythoud C, Rodrigo WWSI, Pasqual G, Rothenberger S, Martinez-Sobrido L, de la Torre JC, et al. Arenavirus Nucleoprotein Targets Interferon Regulatory Factor-Activating Kinase IKK. J Virol (2012) 86:7728-38. doi: 10.1128/ JVI.00187-12

52. Meyer B, Ly H. Inhibition of Innate Immune Responses Is Key to Pathogenesis by Arenaviruses. J Virol (2016) 90:3810-8. doi: 10.1128/ JVI.03049-15

53. Shao J, Huang Q, Liu X, Di D, Liang Y, Ly H. Arenaviral Nucleoproteins Suppress PACT-Induced Augmentation of RIG-I Function To Inhibit Type I Interferon Production. J Virol (2018) 92(13):e00482-18. doi: 10.1128/ JVI.00482-18. JVI.00482-18.

54. Sattler RA, Paessler S, Ly H, Huang C. Animal Models of Lassa Fever. Pathogens (2020) 9:197. doi: 10.3390/pathogens9030197

55. Moskophidis D, Lechner F, Pircher H, Zinkernagel RM. Virus Persistence in Acutely Infected Immunocompetent Mice by Exhaustion of Antiviral Cytotoxic Effector T Cells. Nature (1993) 362:758-61. doi: 10.1038/362758a0

56. Abdel-Hakeem MS. Viruses Teaching Immunology: Role of LCMV Model and Human Viral Infections in Immunological Discoveries. Viruses (2019) 11:106. doi: $10.3390 / \mathrm{v} 11020106$

57. Lazear HM, Pinto AK, Ramos HJ, Vick SC, Shrestha B, Suthar MS, et al. Pattern Recognition Receptor MDA5 Modulates CD8+ T Cell-Dependent Clearance of West Nile Virus From the Central Nervous System. J Virol (2013) 87:11401-15. doi: 10.1128/jvi.01403-13

58. Kandasamy M, Suryawanshi A, Tundup S, Perez JT, Schmolke M, Manicassamy S, et al. RIG-I Signaling Is Critical for Efficient Polyfunctional T Cell Responses During Influenza Virus Infection. PloS Pathog (2016) 12: e1005754. doi: 10.1371/journal.ppat.1005754

59. Marx S, Kümmerer BM, Grützner C, Kato H, Schlee M, Bartok E, et al. RIG-IInduced Innate Antiviral Immunity Protects Mice From Lethal SARS-CoV-2 Infection. bioRxiv (2021) 2021.08.06.455405. doi: 10.1101/2021.08.06.455405

60. Satoh T, Akira S. Toll-Like Receptor Signaling and Its Inducible Proteins. Microbiol Spectr (2016) 4. doi: 10.1128/MICROBIOLSPEC.MCHD-0040-2016

61. Gal-Ben-Ari S, Barrera I, Ehrlich M, Rosenblum K. PKR: A Kinase to Remember. Front Mol Neurosci (2018) 11:480. doi: 10.3389/FNMOL.2018.00480

62. Moresco EMY, Beutler B. LGP2: Positive About Viral Sensing. Proc Natl Acad Sci USA (2010) 107:1261-2. doi: 10.1073/pnas.0914011107

63. Ly H. Differential Immune Responses to New World and Old World Mammalian Arenaviruses. Int J Mol Sci (2017) 18:1040. doi: 10.3390/ ijms 18051040

64. Mantlo E, Paessler S, Huang C. Differential Immune Responses to Hemorrhagic Fever-Causing Arenaviruses. Vaccines (2019) 7:138. doi: 10.3390/vaccines7040138

65. Cuevas CD, Lavanya M, Wang E, Ross SR. Junin Virus Infects Mouse Cells and Induces Innate Immune Responses. J Virol (2011) 85:11058-68. doi: 10.1128/JVI.05304-11

66. Cuevas CD, Ross SR. Toll-Like Receptor 2-Mediated Innate Immune Responses Against Junín Virus in Mice Lead to Antiviral Adaptive Immune 
Responses During Systemic Infection and do Not Affect Viral Replication in the Brain. J Virol (2014) 88:7703-14. doi: 10.1128/JVI.00050-14

67. Huang C, Kolokoltsova OA, Mateer EJ, Koma T, Paessler S. Highly Pathogenic New World Arenavirus Infection Activates the Pattern Recognition Receptor Protein Kinase R Without Attenuating Virus Replication in Human Cells. J Virol (2017) 91:e01090-17. doi: 10.1128/ JVI.01090-17

68. Platanias LC. Mechanisms of Type-I- and Type-II-Interferon-Mediated Signalling. Nat Rev Immunol 2005 5:5 (2005) 5:375-86. doi: 10.1038/nri1604

69. Lee AJ, Ashkar AA. The Dual Nature of Type I and Type II Interferons. Front Immunol (2018) 8:2061. doi: 10.3389/FIMMU.2018.02061

70. Castro F, Cardoso AP, Gonçalves RM, Serre K, Oliveira MJ. InterferonGamma at the Crossroads of Tumor Immune Surveillance or Evasion. Front Immunol (2018) 9:847. doi: 10.3389/FIMMU.2018.00847

71. Odendall C, Kagan JC. The Unique Regulation and Functions of Type III Interferons in Antiviral Immunity. Curr Opin Virol (2015) 12:47. doi: 10.1016/ J.COVIRO.2015.02.003

72. Pien GC, Nguyen KB, Malmgaard L, Satoskar AR. Biron CA. A Unique Mechanism for Innate Cytokine Promotion of T Cell Responses to Viral Infections. J Immunol (2002) 156:1138-42. doi: 10.4049/jimmunol. 169.10 .5827

73. Asper M, Sternsdorf T, Hass M, Drosten C, Rhode A, Schmitz H, et al. Inhibition of Different Lassa Virus Strains by Alpha and Gamma Interferons and Comparison With a Less Pathogenic Arenavirus. J Virol (2004) 78:3162. doi: 10.1128/JVI.78.6.3162-3169.2004

74. Baize S, Pannetier D, Faure C, Marianneau P, Marendat I, Georges-Courbot $\mathrm{MC}$, et al. Role of Interferons in the Control of Lassa Virus Replication in Human Dendritic Cells and Macrophages. Microbes Infect (2006) 8:1194-202. doi: 10.1016/J.MICINF.2006.02.002

75. Kolokoltsova OA, Yun NE, Poussard AL, Smith JK, Smith JN, Salazar M, et al. Mice Lacking Alpha/Beta and Gamma Interferon Receptors Are Susceptible to Junin Virus Infection. J Virol (2010) 84:13063-7. doi: 10.1128/JVI.01389-10

76. Huang C, Walker AG, Grant AM, Kolokoltsova OA, Yun NE, Seregin AV, et al. Potent Inhibition of Junín Virus Infection by Interferon in Murine Cells. PloS Negl Trop Dis (2014) 8:e2933. doi: 10.1371/JOURNAL.PNTD.0002933

77. Sahin M, Remy MM, Merkler D, Pinschewer DD. The Janus Kinase Inhibitor Ruxolitinib Prevents Terminal Shock in a Mouse Model of Arenavirus Hemorrhagic Fever. Microorganisms (2021) 9:564. doi: 10.3390/ MICROORGANISMS9030564

78. Xing J, Chai Z, Ly H, Liang Y. Differential Inhibition of Macrophage Activation by Lymphocytic Choriomeningitis Virus and Pichinde Virus Is Mediated by the Z Protein N-Terminal Domain. J Virol (2015) 89:12513-7. doi: 10.1128/JVI.01674-15

79. Whitmire JK, Tan JT, Whitton L. Interferon $-\gamma$ Acts Directly on CD8+ T Cells to Increase Their Abundance During Virus Infection. J Exp Med (2005) 201:1053-9. doi: $10.1084 /$ jem.20041463
80. Sommereyns C, Paul S, Staeheli P, Michiels T. IFN-Lambda (IFN- $\lambda$ ) Is Expressed in a Tissue-Dependent Fashion and Primarily Acts on Epithelial Cells In Vivo. PloS Pathog (2008) 4:e1000017. doi: 10.1371/journal. ppat. 1000017

81. Walker CM, Rawls WE, Rosenthal KL. Generation of Memory Cell-Mediated Immune Responses After Secondary Infection of Mice With Pichinde Virus. J Immunol (Baltimore Md: 1950) (1984) 132:469-74.

82. Rosenthal KL, Steiner C, Rawls WE, Walker CM. Studies Concerning the Relationship of Pichinde Virus-Induced Natural Killer Cells and Cytotoxic T Lymphocytes. Med Microbiol Immunol (1986) 175:133-6. doi: 10.1007/ BF02122433

83. Vargas-Cortes M, O’Donnell CL, Maciaszek JW, Welsh RM. Generation of "Natural Killer Cell-Escape" Variants of Pichinde Virus During Acute and Persistent Infections. J Virol (1992) 66:2532-5. doi: 10.1128/jvi.66.4.25322535.1992

84. Fan L, Briese T, Lipkin WI. Z Proteins of New World Arenaviruses Bind RIG-I and Interfere With Type I Interferon Induction. J Virol (2010) 84:1785-91. doi: 10.1128/JVI.01362-09

85. Xing J, Ly H, Liang Y. The Z Proteins of Pathogenic But Not Nonpathogenic Arenaviruses Inhibit RIG-I-Like Receptor-Dependent Interferon Production. J Virol (2015) 89:2944-55. doi: 10.1128/JVI.03349-14

86. Fredericksen BL, Keller BC, Fornek J, Katze MG, Gale M. Establishment and Maintenance of the Innate Antiviral Response to West Nile Virus Involves Both RIG-I and MDA5 Signaling Through IPS-1 †. J Virol (2008) 82:609-16. doi: 10.1128/JVI.01305-07

87. Nasirudeen AMA, Wong HH, Thien P, Xu S, Lam K-P, Liu DX. RIG-I, MDA5 and TLR3 Synergistically Play an Important Role in Restriction of Dengue Virus Infection. PloS Negl Trop Dis (2011) 5:e926. doi: 10.1371/journal. pntd.0000926

Conflict of Interest: The authors declare that the research was conducted in the absence of any commercial or financial relationships that could be construed as a potential conflict of interest.

Publisher's Note: All claims expressed in this article are solely those of the authors and do not necessarily represent those of their affiliated organizations, or those of the publisher, the editors and the reviewers. Any product that may be evaluated in this article, or claim that may be made by its manufacturer, is not guaranteed or endorsed by the publisher.

Copyright $\odot 2021$ Brisse, Huang, Rahman, Di, Liang and Ly. This is an open-access article distributed under the terms of the Creative Commons Attribution License (CC BY). The use, distribution or reproduction in other forums is permitted, provided the original author(s) and the copyright owner(s) are credited and that the original publication in this journal is cited, in accordance with accepted academic practice. No use, distribution or reproduction is permitted which does not comply with these terms. 